\title{
Public and Private Water: Analysis of Consumer's Behaviors
}

\author{
Gianluca Senatore
}

\author{
Sapienza Università di Roma, \\ Piazzale Aldo Moro, 5, 00185 \\ Roma RM, Italy
}

DOI: https://doi.org/10.36941/ajis-2021-0147

\begin{abstract}
In this work we have pointed out how the use of bottled water affects our economic, social and environmental system comparing this phenomenon to the use of public water. Trough a detaild analysis and a social experiment about preferences between public and private water (plastic bottled), we have tried to understand deeply how and why the phenomenon of bottled water is always increasing in Italy if we compare it to what happens in other countries.
\end{abstract}

Keywords: water, plastic bottles, consumer preferences, sustainability

\section{Introduzione}

Il consumo non può essere considerato solo la manifestazione di un comportamento economico, ma è anche l'espressione diretta di fattori psicologici, sociologici, etici e fisiologici, pertanto per l'analisi delle scelte del consumatore non è sufficiente partire unicamente da un approccio economico. A questo proposito, adottare un approccio interdisciplinare che tenga conto della teoria dell'organizzazione, della scienza amministrativa classica, della psicologia, della sociologia e dell'antropologia (Cabiddu, 2007), può restituire un'analisi più approfondita del fenomeno, considerando anche le dimensioni sociali che possono incidere sui comportamenti di consumo. Per il seguente lavoro ci siamo concentrati nello specifico sul comportamento dei consumatori rispetto l'utilizzo di acqua pubblica e privata, quindi in che modo le dimensioni sociali possono influire su tali scelte di consumo.

Il consumo di acqua in bottiglia si è diffuso in Italia all'inizio degli anni ' 70 ed è cresciuto notevolmente a seguito del cambiamento delle abitudini del consumatore, influenzate da uno stimolo sempre maggiore ad utilizzare acqua in bottiglia, condizionato sia da fenomeni sociali che da una modifica della percezione dell'acqua, passata dall'essere una bevanda essenziale ad una fonte di salute e bellezza (Torretta, 2013).

$\mathrm{Al}$ fine di comprendere meglio quali sono le dimensioni e gli aspetti che influenzano la scelta del consumatore nel preferire acqua privata (imbottigliata) rispetto a quella pubblica e viceversa, il seguente lavoro illustrerà le fasi di una ricerca svolta presso la sede del dipartimento di Comunicazione e ricerca sociale della Sapienza e presso la Città Universitaria nel novembre 2017. L'intero progetto è stato ideato, costruito e realizzato nell'ambito della cattedra di Comportamenti collettivi e sostenibilità socio-ambientale e ha vito il coinvolgimento di molti studenti del corso ${ }^{1}$. Il

${ }^{1}$ Gli studenti che hanno partecipato alla realizzazione del progetto sono: Franco Galato, Dario Germani, Roberta Iacobucci, Yuri Ciro Mennella, Eugenia Murabito, Marco Paluzzi, Claudia Sesto, Michela Cavagnuolo, Alessandra De Carlo, Francesca Sparice, Ilaria Nappi. 
questionario è stato svolto inoltre con il supporto di un esperimento sociale, con l'obiettivo di indagare meglio gli atteggiamenti nei confronti del consumo di acqua pubblica e privata.

\section{Il Consumo Dell'acqua e i Suoi Impatti Sull'ambiente}

Il consumo di acqua in bottiglia si è diffuso in Italia all'inizio degli anni ' 70 , per poi crescere notevolmente anche a causa del cambiamento delle abitudini del consumatore. Il consumatore ha infatti avuto uno stimolo sempre maggiore ad utilizzare acqua in bottiglia, dovuto spesso a fenomeni sociali, come l'attribuzione di tale tipo di consumo ad un simbolo di status, inoltre i crescenti investimenti in campagne pubblicitarie da parte di multinazionali, così come è successo per altri prodotti, ha modificato la percezione dell'acqua dall'essere una bevanda essenziale e basilare a fonte di salute e bellezza (Torretta, 2013). La conseguenza di questo cambiamento nei comportamenti di consumo è ricaduta sull'ambiente, l'impatto ambientale dovuto alla filiera dell'acqua, infatti è molto significativo se si considera l'utilizzo di bottiglie di plastica, il consumo di olio necessario per la produzione delle bottiglie, l'emissione dei veicoli che trasportano le bottiglie o gli imballaggi di plastica non riciclata (Torretta, 2013).

Nel 2018 in Italia venivano utilizzati tra i 7,2 e gli 8,4 miliardi di bottiglie di plastica, infatti il 9o$95 \%$ delle acque era imbottigliata in contenitori di plastica e il 5-10\% in contenitori in vetro (Legambiente, 2018). Tale fenomeno ha avuto impatti positivi in termini di numero di dipendenti e fatturato per le società di bevande, ma considerando la durata delle bottiglie d'acqua in plastica e gli impatti dell'industria dell'acqua minerale, l'aspetto ambientale risente di tali impatti in modo rilevante. Nello specifico, attualmente, per imbottigliare i circa 12 miliardi di acqua minerale utilizzata annualmente dagli italiani, sono utilizzate 350 mila tonnellate di PET, e vengono consumate 665 mila tonnellate di petrolio, con una conseguente emissione di gas serra di circa 245.679 tonnellate di CO2 equivalente (Legambiente, 2018). Il trasporto di acqua minerale ha inoltre un impatto rilevante sull'inquinamento atmosferico in quanto le bottiglie percorrono molti $\mathrm{km}$ prima di arrivare ai consumatori, e solo il $18 \%$ del totale delle merci in bottiglia viene trasportato su rotaie, infine, in Europa solo un terzo delle bottiglie usate viene poi riciclato (Torretta, 2013). Nel grafico che segue sono riportati alcuni dati riguardo le emissioni giornaliere di $\mathrm{CO}_{2}$ da parte dei camion necessari per il trasporto di acque in bottiglia, nonché i costi che tale operazione richiede.

\begin{tabular}{|c|c|c|c|c|c|}
\hline BRAND ACQUA & $\begin{array}{c}\text { Emissioni } \\
\text { giornaliera di } \\
\mathrm{Co} 2 \text { da parte } \\
\text { di un solo } \\
\text { camion }(\mathrm{Kg})\end{array}$ & $\begin{array}{l}\text { Emissioni } \\
\text { giomaliera di } \\
\mathrm{Co} 2 \text { per } \\
300000 \\
\text { camion }(\mathrm{Kg})\end{array}$ & $\begin{array}{l}\text { Costo al } \\
\text { giomo } \\
\text { (euro) }\end{array}$ & $\begin{array}{l}\text { Costo al } \\
\text { mese } \\
\text { (euro) }\end{array}$ & $\begin{array}{l}\text { Costo } \\
\text { annuale } \\
\text { (euro) }\end{array}$ \\
\hline San Pellegrino & 13675 & 4102500000 & $3.256 €$ & $81.400 €$ & $976.800 €$ \\
\hline San Benedetto & 12383 & 3714900000 & $2.949 €$ & $73.725 €$ & $884.700 €$ \\
\hline Fonti di Vinadio & 16286 & 4885800000 & $3.878 €$ & $96.950 €$ & $1.163 .400 €$ \\
\hline $\begin{array}{c}\text { Norda } \\
\text { Gaudiniello }\end{array}$ & 13276 & 3982800000 & $3.204 €$ & $80.100 €$ & $961.200 €$ \\
\hline $\begin{array}{c}\text { Gruppo Co.Ge. Di } \\
\text { (Uliveto- } \\
\text { Rocchetta) }\end{array}$ & 10536 & 3160800000 & $2.509 €$ & $62.725 €$ & $752.700 €$ \\
\hline Ferrarelle & 11737 & 3521100000 & $2.795 €$ & $83.850 €$ & $1.006 .200 €$ \\
\hline Totale & 77893 & 23367900000 & $\begin{array}{c}18.5910 \\
€\end{array}$ & $\begin{array}{c}394.900 \\
€\end{array}$ & $4.738 .800 €$ \\
\hline 11 & 11 & II & II & 11 & II \\
\hline $\begin{array}{c}\mathrm{Km} * \mathrm{~kg} \mathrm{di} \\
\text { emissioni (1.2) } \\
{ }^{*} 98 \% \text { di Co2 }\end{array}$ & & & & & \\
\hline $\begin{array}{l}\mathrm{Km} / \text { litri*costo } \\
\text { gasolio }\end{array}$ & & & & & \\
\hline $\begin{array}{l}\text { Totale emissioni } \\
\text { Co2 (camion e } \\
\text { produzione } \\
\text { plastica) }\end{array}$ & 24567900000 & & & & \\
\hline $\begin{array}{c}\text { Manutenzione } \\
\text { annua per ciascun } \\
\text { camion }\end{array}$ & $5700 €$ & & & & \\
\hline
\end{tabular}

Figura 1: Brand acqua e emissioni

Fonte: https://www.sanpellegrino.com/it/it, https:/www.cogedi.it/it/index.html, https://www.santanna.it/, https://www.ferrarelle.it/, https://www.sanbenedetto.it/it/home/ 
Lo schema che segue illustra inoltre il lungo percorso che una bottiglia in PET deve percorrere prima di essere distribuita ai consumatori. Un indotto di produzione che coinvolge diversi settori e incide di conseguenza sull'ambiente su più fronti.

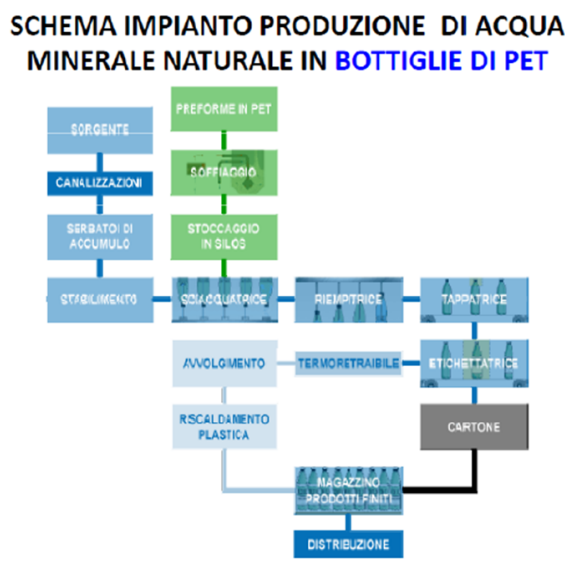

Figura 2: Schema impianto produzione di acqua minerale naturale in bottiglie di PET Fonte: https://www.acque.net/

Non solo la produzione delle bottiglie d'acqua, ma anche il loro smaltimento è da considerarsi dannoso per l'ambiente. Il PET è una plastica non biodegradabile (Shah et al., 2008) e per tale motivo lo smaltimento dopo il suo utilizzo ha un enorme impatto ambientale, al punto che tale materiale è da considerarsi il più presente tra i rifiuti solidi urbani (de Mello, Pezzin and Amico, 2009). Le bottiglie in PET utilizzate per uso domestico vengono raccolte, compresse e imballate per essere poi trasportate agli impianti di riciclaggio, le restanti bottiglie, dopo la selezione, vengono triturate, pulite e trasformate in pellet per il riciclaggio (Al-Salem, Lettieri and Baeyens, 2009). Il riciclaggio dei materiali in PET utilizzati per l'imballaggio, non è stato possibile per molto tempo in quanto non si avevano delle conoscenze riguardo la contaminazione dei polimeri di imballaggio durante il primo utilizzo o la raccolta e non si conoscevano inoltre le efficienze di decontaminazione dei processi di riciclaggio (Torretta, 2013). Diversamente, oggi, sono stati sviluppati avanzati processi di decontaminazione, al punto di ottenere materiali PET vergini dopo il riciclo (Welle, 2011). Nonostante ciò, c'è sempre una quantità di materiale che però non può essere riciclato e non è recuperabile, quindi destinato ad essere smaltito in discarica $\mathrm{e}$ ad aumentare la mole di rifiuti in plastica in circolazione (Torretta, 2013).

Nel 2018 l'Italia era solamente quinta in Europa per la quantità e il tasso di riciclaggio dei rifiuti di imballaggi in plastica (Parlamento Europeo, 2018) (Fig. 3).

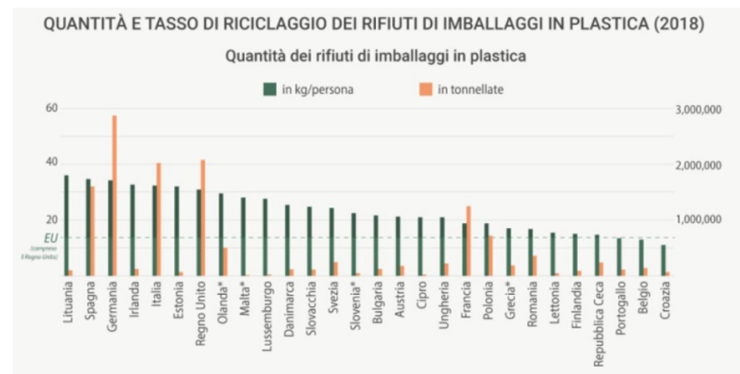

Figura 3: Quantità e tasso di riciclaggio dei rifiuti di imballaggi in plastica Fonte: Parlamento Europeo 
La produzione di plastica è aumentata in maniera esponenziale in pochi decenni, passando dal milione e mezzo di tonnellate nel 1950 ai 359 milioni di tonnellate nel 2018, con una conseguente impennata dei rifiuti di plastica (Parlamento Europeo, 2018). Recentemente si è assistito invece ad un significativo calo di produzione, nella prima metà del 2020, a causa del Covid-19, ma la produzione è ripresa nuovamente nella seconda metà dell'anno.

Dall'immagine che segue (fig. 4) inoltre, si può notare come la principale produzione di plastica in UE è quella degli imballaggi, quali appunto bottiglie di plastica o buste di plastica, seguito da materiali edili e da costruzione. Per quanto riguarda invece il trattamento di tali rifiuti in plastica, il $42.6 \%$ di essi viene recuperato per la produzione di energie, il $24.9 \%$ interrato e solo il $32.5 \%$ viene riciclato. Recenti rapporti hanno stimato una emissione di 850 milioni di tonnellate di gas serra a livello globale - solo nel 2017 - a causa della produzione e dell'incenerimento della plastica, emissioni che potrebbero salire addirittura a 2,8 miliardi di tonnellate entro il 2050, a meno che non vengano istituiti metodi di riciclaggio più efficaci (Parlamento Europeo, 2018).

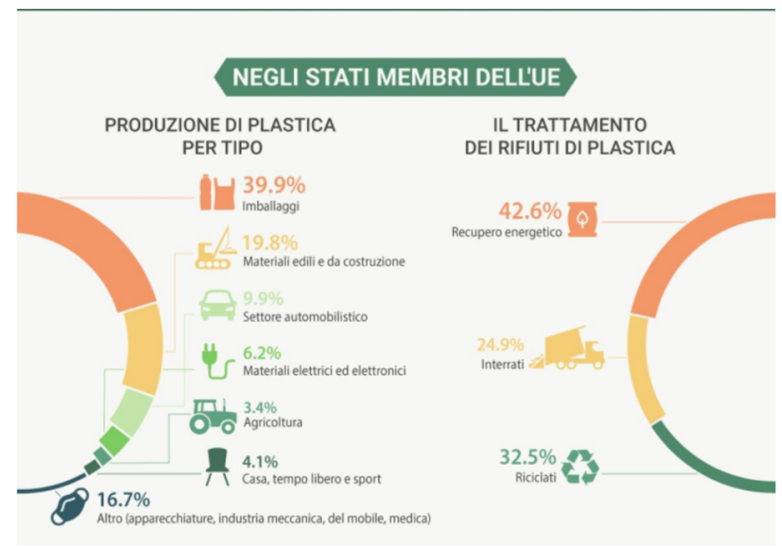

Figura 4: Produzione di plastica per tipo e trattamento dei rifiuti in plastica in UE Fonte: Parlamento Europeo

Nello specifico l'Italia, stando ai dati del 2019, risulta sul podio tra i paesi mondiali che più consumano acque minerali e confezionate in bottiglia. Più del $60 \%$ degli 11 miliardi di bottiglie che vengono consumate in Italia ogni anno non vengono riciclate, e 7 miliardi di contenitori in PET rischiano di essere dispersi nell'ambiente e nei mari. L'impatto negativo sull'ambiente aumenta se si considerano anche le emissioni di gas serra impiegate per la produzione delle bottiglie non riciclate, che nel nostro Paese è pari a 850 mila tonnellate di $\mathrm{CO}_{2}$ equivalenti (Greenpeace, 2021).

Gli impatti negativi sull'ambiente che ne derivano non hanno contribuito comunque a ridurre l'utilizzo dell'acqua in bottiglia da parte dei consumatori, che la preferiscono nella maggior parte dei casi all'acqua potabile, infatti nonostante l'Italia sia un paese caratterizzato per un'abbondanza di acqua di sorgente e di falda, con buone qualità organolettiche, come accennato precedentemente, è tra i primi in Europa e secondo al mondo per consumo pro-capite di acqua minerale. Le aziende leader del mercato nazionale delle acque minerali sono San Benedetto, Nestlè-San Pellegrino, e Sant'Anna, per quanto riguarda le bibite invece il mercato è guidato da Coca Cola, San Benedetto e Nestlè-San Pellegrino. In particolare, nel 2019, 11 miliardi di litri di acqua confezionata e utilizzata in Italia è stata venduta in bottiglie monouso in PET, oltre 2 miliardi in bottiglie in vetro e 0,3 miliardi in altre tipologie di contenitori, aggiungendo anche il consumo di bevande diverse dalle acque minerali, si raggiugono 13,7 miliardi di litri di bevande confezionate in PET. Il riciclo effettivo di questa enorme mole di materiali in plastica, risulta di poco inferiore al $40 \%$, dunque quasi 280 mila delle 460 mila tonnellate di bottiglie in PET consumate ogni anno, non viene riciclata, mentre l'equivalente di 7 
miliardi di bottiglie da un litro e mezzo viene incenerita nei termovalorizzatori e nei cementifici, dispersa nell'ambiente o smaltita in discarica (Greenpeace, 2021). Le cause per cui, nonostante gli importanti effetti negativi sull'ambiente, gli italiani continuano a preferire acqua minerale in bottiglia, possono essere determinate da diversi fattori, come le numerose campagne pubblicitarie che condizionano i comportamenti dei consumatori, la mancanza di chiarezza dei legislatori che contribuisce ad aumentare l'incertezza del cittadino sulle questioni legate all'impatto del monouso o la percezione dei consumatori secondo cui l'acqua in bottiglia sia migliore e più controllata rispetto a quella del rubinetto.

Ma qual è la reale differenza tra l'acqua imbottigliata e l'acqua pubblica (di acquedotto)? L'acqua in bottiglia, anche meglio conosciuta come acqua minerale è un tipo di acqua sorgiva che viene commercializzata appunto in bottiglia, e può essere venduta, in Italia, con tale dicitura solo se risponde ai criteri di legge stabiliti dal D.lgs. 8/10/2011 n. 176 (attuazione della direttiva 2009/54/CE). Il consumo di acqua minerale in bottiglia è triplicato dal 1985, pertanto la legge ha imposto controlli da parte dell'autorità sanitaria alla sorgente, con una cadenza almeno quadrimestrale; all'impianto di imbottigliamento, con frequenza mensile o settimanale; ai depositi all'ingrosso e alla vendita al dettaglio da parte delle ASL con cadenza diversa in base alla regione di riferimento. Inoltre, vengono effettuati controlli chimici e microbiologici da parte dell'azienda di imbottigliamento e un controllo annuale completo da parte di un laboratorio autorizzato. Le acque in bottiglia, dunque, subiscono numerosi controlli, ma le analisi svolte sulle acque di acquedotto sono molto più frequenti e con soglie di sicurezza spesso più elevate. I controlli sulle acque potabili comunali vengono effettuati ogni giorno e quindi possono essere considerate più controllate delle acque in bottiglia, le quali vengono imbottigliate così come risultano alla fonte, mentre l'acqua di rubinetto viene potabilizzata con un processo di clorazione al fine di eliminare i rischi di contaminazione batterica. La presenza di cloro all'interno dell'acqua di rubinetto può essere proprio quella che dà un gusto non gradevole e portare il consumatore a preferire l'acqua in bottiglia. Il consumo maggiore di acqua in bottiglia, soprattutto in Italia, pare essere strutturale, influenzato dai cambiamenti socio-economici. Tali abitudini, ormai consolidate, portano alla conseguenza che l'acqua pubblica oggi, su cui negli ultimi anni vi sono stati importanti investimenti per consentire elevati livelli di accessibilità e garantire il diritto all'acqua potabile a tutti i cittadini, viene utilizzata principalmente per usi domestici. Ciò accade in quanto l'acqua in bottiglia viene considerata più sicura rispetto a quella del rubinetto, ma soffermandosi sulle etichette di alcune marche si può notare come ciò non sia vero, bensì confrontando i parametri che caratterizzano un'acqua minerale con quelli dell'acqua di rete, emerge che la qualità delle acque minerali non sempre raggiunge gli stessi livelli di quella potabile, aspetto confermato dal fatto che la legge tollera nelle acque imbottigliate una concentrazione di sostanze nocive maggiore rispetto alle acque di acquedotto (fig. 5).

\begin{tabular}{|l|c|c|}
\hline Elemento & $\begin{array}{c}\text { Limite acquedotto } \\
(\mathrm{mg} / \mathrm{lt})\end{array}$ & $\begin{array}{c}\text { Limite acque minerali } \\
(\mathrm{mg} / \mathrm{lt})\end{array}$ \\
\hline antimonio & 5 & Nessun limite \\
\hline arsenico & 10 & 50 \\
\hline boro & 1 & 5 \\
\hline nichel & 20 & Nessun limite \\
\hline vanadio & 50 & Nessun limite \\
\hline alluminio & 200 & Nessun limite \\
\hline ferro & 200 & Nessun limite \\
\hline manganese & 50 & 2000 \\
\hline ione fluoruro & 1,5 & Nessun limite \\
\hline
\end{tabular}

Non si trovano indicati in etichetta i risultati dei seguenti elementi:

cianuri, fenoli, tensioattivi,oli minerali, idrocarburi pesticidi, policlorurati, bario, cadmio, cromo, mercurio, piombo, rame, selenio.

Figura 5: Elementi aggiuntivi acqua

Fonte: www.acqua.net 
La qualità delle acque destinate al consumo in Italia deve essere quindi conforme ad una serie di parametri microbiologici (Decreto legislativo 31/2001, parte A), chimici (parte B) e parametri indicatori (parte C), i quali non sono direttamente correlabili a rischi per la salute umana, ma fungono da indicatori capaci di evidenziare eventuali modifiche della qualità delle acque, assicurata per l' $85 \%$ da acque sotterranee, protette da un sistema esteso di controlli da parte di chi gestisce i servizi idrici e da parte delle Autorità Sanitarie. I parametri chimici individuati nel Decreto citato precedentemente, includono tutti i parametri della Direttiva 98/83/CE (Comunità Europea, 1998), di cui adotta gli stessi valori, a volte con criteri anche più stringenti, in esso inoltre sono stati inseriti ulteriori parametri tenendo conto del principio della sussidiarietà, considerando le caratteristiche delle risorse idriche e dei sistemi idro-potabili dell'intero territorio nazionale.

\section{Analisi della Scelta tra Acqua Pubblica e Privata}

Alla luce di quanto detto precedentemente sul maggiore uso di acqua in bottiglia, rispetto all'acqua potabile di rubinetto, a novembre 2017 è stato svolto un esperimento presso il Dipartimento di Comunicazione e Ricerca sociale dell'Università degli studi di Roma La Sapienza, sito in via Salaria ${ }^{2}$. Lo scopo dell'esperimento è stato quello di evidenziare come la scelta tra acqua pubblica e privata non è dovuta alle proprietà dell'acqua pubblica rispetto a quella privata, infatti l'evidente mancanza di differenze tra acqua pubblica e privata è l'ipotesi dalla quale si è partiti per definire l'esperimento, mentre l'unità d'analisi scelta è stata l'individuo, e la popolazione a cui si è fatto riferimento è stata quella dei cittadini di Roma, selezionati attraverso un campionamento casuale, inizialmente di 100 casi per poi estendersi a 150 in fase di ricerca.

Per ciò che concerne lo strumento di rilevazione scelto invece, è stato svolto un questionario prima e dopo l'esperimento.

Nel procedere per la stesura delle domande del questionario, sono state prese in considerazione varie dimensioni, variabili ed indicatori. Nello specifico le dimensioni e le variabili selezionate sono state le seguenti:

- Socio-anagrafiche: genere, anno di nascita, città/paese di provenienza, luogo di residenza/domicilio attuale;

- Status: titolo di studio, condizione occupazionale, stato civile;

- Percezione nei confronti dell'acqua pubblica e privata: quale acqua usa quando beve, per quali ragioni preferisce bere acqua in bottiglia, per quali ragioni preferisce bere acqua dal rubinetto;

- Capitale culturale: tipo di acqua utilizzata nella famiglia di provenienza, per quali ragioni si utilizza acqua in bottiglia, per quali ragioni si utilizza acqua dal rubinetto;

- Atteggiamento: atteggiamento acqua privata, atteggiamento acqua pubblica;

- Abitudine: abitudini di comportamento quando si è fuori casa.

Dopo aver posto le domande, è stato chiesto agli intervistati di partecipare all'esperimento sociale e, se d'accordo, è stato chiesto di procedere col rispondere agli ultimi quesiti, per verificare eventuali modifiche dell'atteggiamento dopo l'esperimento. In particolare, le dimensioni che sono state nuovamente oggetto di domanda sono state quelle che interessavano appunto l'atteggiamento nei confronti dell'uso di acqua privata e dell'uso di acqua pubblica.

Per la raccolta dei dati si è proceduto quindi con un'intervista standardizzata, condotta con l'ausilio di un questionario cartaceo come supporto per l'esperimento sociale, presso Via Salaria 113 e la Città Universitaria della Sapienza. Il questionario è stato somministrato con la modalità face to

2 L'esperimento è stato svolto durante il corso di Comportamenti collettivi e sostenibilità socioambientale, condotto dagli studenti del corso: Franco Galato, Dario Germani, Roberta Iacobucci, Yuri Ciro Mennella, Eugenia Murabito, Marco Paluzzi, Claudia Sesto, Michela Cavagnuolo, Alessandra De Carlo, Francesca Sparice, Ilaria Nappi. 
face.

Per l'analisi dei dati invece è stato utilizzato il software SPSS (Statistical Package for Social Science), ed è stata svolta nello specifico un'analisi monovariata, successivamente una bivariata e infine è stato fatto un confronto fra gli intervistati e il loro background culturale, e un'analisi dell'atteggiamento degli intervistati nei confronti dell'acqua pubblica e dell'acqua privata, prima e dopo l'esperimento. Di seguito riporteremo i risultati della ricerca, con il supporto di alcuni grafici.

I soggetti intervistati sono stati sia maschi $(53 \%)$ che femmine $(47 \%)$ in maniera equilibrata, i quali per la maggior parte provenivano dal Centro (74\%), per il $4 \%$ dal Nord e per il $22 \%$ dal Sud e dalle Isole (fig. 6).

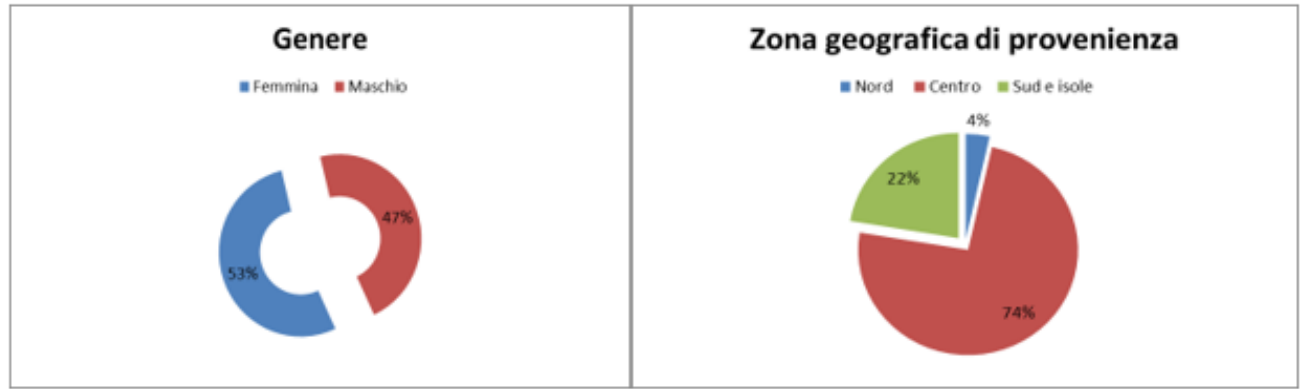

Figura 6: Genere e zona geografica

La figura 7 illustra invece il tipo di acqua bevuta dagli intervistati, che per la maggior parte dei casi utilizza acqua in bottiglia ( $57 \%$ ), rispetto ad un $43 \%$ che utilizza acqua del rubinetto.

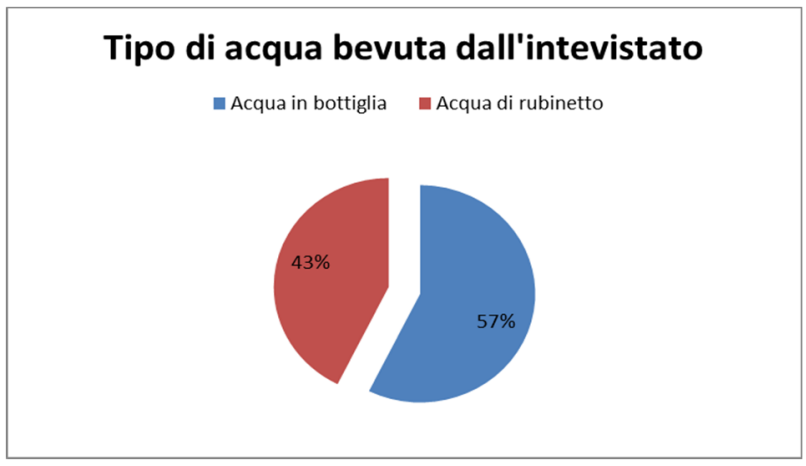

Figura 7 Tipo di acqua bevuta dall'intervistato

Per comprendere invece i motivi che spingono gli intervistati a preferire l'acqua in bottiglia piuttosto che quella pubblica, la figura 8 evidenzia come il $46 \%$ degli intervistati preferisca l'acqua in bottiglia perché ha un sapore più buono, il $38 \%$ la considera più controllata rispetto a quella del rubinetto, il $12 \%$ invece perché l'etichetta dà indicazioni sulla sua composizione, e infine un $4 \%$ la utilizza per motivi di salute, perché prescritta dal medico. L'acqua pubblica invece è preferita dal $41 \%$ degli intervistati perché è gratuita, dal 38\% perché l'approvvigionamento è più comodo, dal $10 \%$ perché si ritiene che il sapore sia più buono, dal $5 \%$ perché non è soggetta a processi industriali e infine solo il $6 \%$ la considera più sicura perché consapevole che viene controllata giornalmente. 


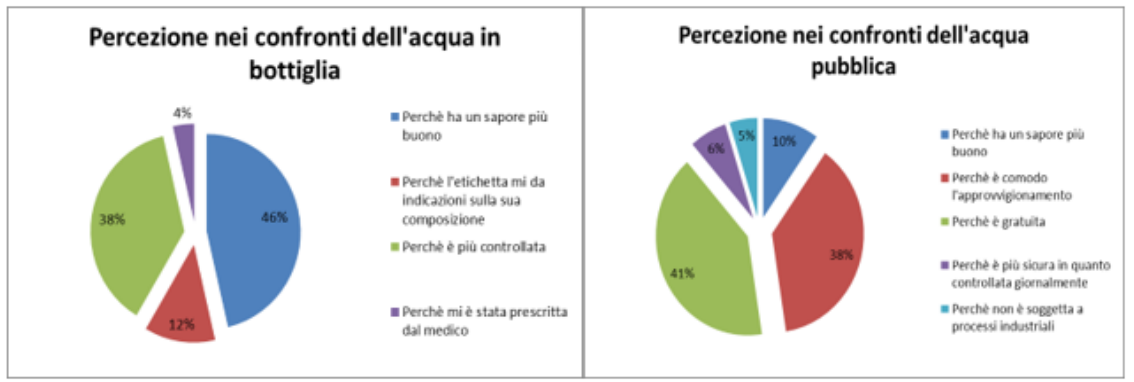

Figura 8: Percezione nei confronti dell'acqua in bottiglia e dell'acqua pubblica

Prendendo in considerazione invece il background culturale degli intervistati, di seguito sono riportati alcuni grafici illustrativi che aiutano meglio a comprendere quale sia stata l'influenza da parte della famiglia di origine riguardo la scelta tra acqua pubblica e privata.

La figura 9 evidenza come il $62 \%$ delle famiglie di origine degli intervistati beve acqua in bottiglia, rispetto ad un $38 \%$ che invece utilizza acqua di rubinetto.

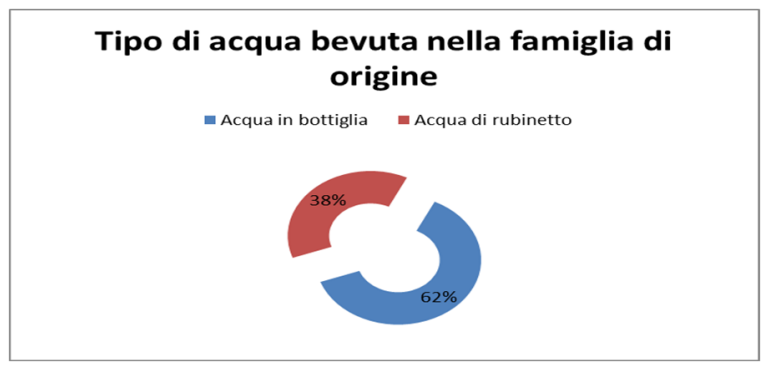

Figura 9: Tipo di acqua bevuta nella famiglia di origine

I motivi per cui le famiglie di origine preferiscono l'acqua in bottiglia (fig. 10) è principalmente per il sapore più gradevole $(45 \%)$ e perché considerata più controllata $(40 \%)$, il $12 \%$ invece la utilizza perché l'etichetta dà informazioni sulla sua composizione e il $3 \%$ perché prescritta dal medico. L'acqua pubblica è preferita invece dalle famiglie di origine degli intervistati (fig. 10) per il 50\% dei casi perché è comodo l'approvvigionamento, per il $25 \%$ perché è gratuita, il $16 \%$ la considera più buona rispetto a quella in bottiglia, il $7 \%$ perché non è sottoposta a processi industriali e infine solo il $2 \%$ la considera più sicura in quanto è controllata giornalmente.

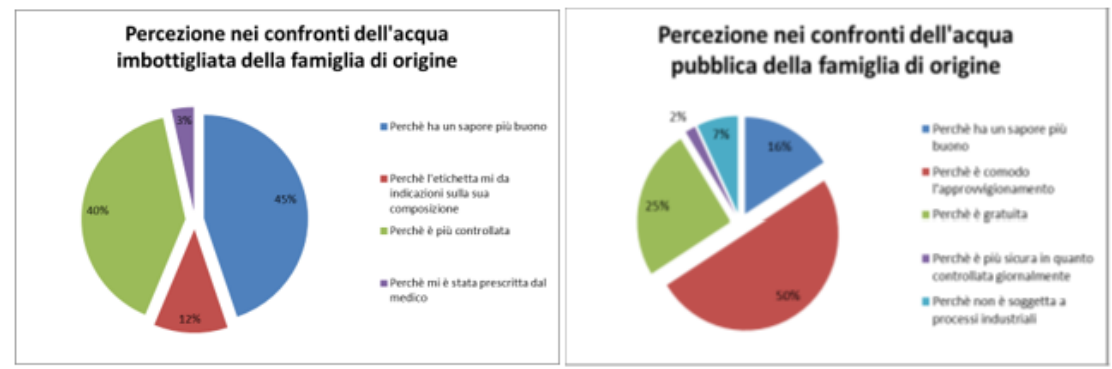

Figura 1o: Percezione nei confronti dell'acqua imbottigliata e dell'acqua pubblica della famiglia di origine 
Da una successiva analisi bivariata, eseguita per verificare la relazione esistente tra il contesto culturale e il tipo di acqua bevuta, è emerso che non si rilevano differenze statistiche tra le due variabili, quindi sono indipendenti: l'utilizzo di un tipo di acqua da parte degli intervistati non dipende dal tipo di acqua bevuta nella famiglia di origine, come esemplifica la figura 11.

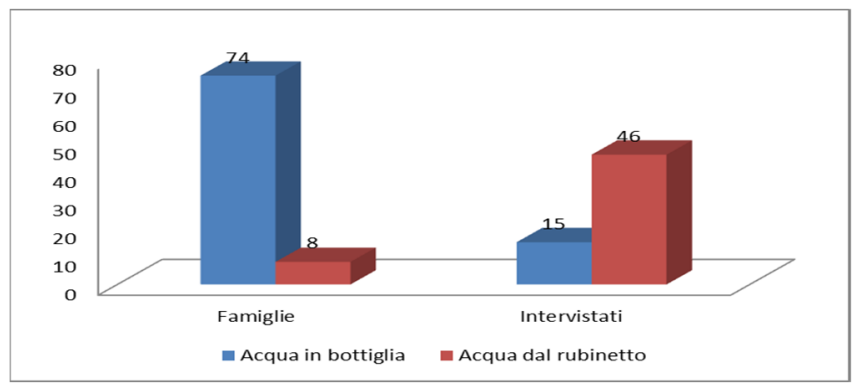

Figura 11: Tipo di acqua utilizzata dagli intervistati e dalle famiglie

Volendo indagare più a fondo in base a cosa viene scelta un'acqua in bottiglia piuttosto che un'altra, inoltre, dalle interviste è emerso che il 34\% la sceglie in base al prezzo, il $22 \%$ casualmente o per le proprietà organolettiche che possiede, il $18 \%$ per il gusto e nel $2 \%$ dei casi invece influisce il packaging della bottiglia e la pubblicità con la quale viene promossa (fig. 12).

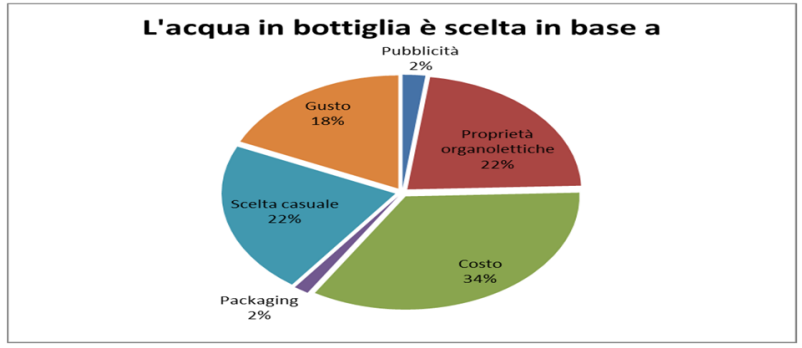

Figura 12: L'acqua in bottiglia è scelta in base a

Successivamente, al fine di comprendere l'atteggiamento degli intervistati nei confronti dell'acqua imbottigliata e dell'acqua pubblica, prima dell'esperimento, sono state poste una serie di domande alle quali poter attribuire un grado di disaccordo o di accordo (fig. 13).
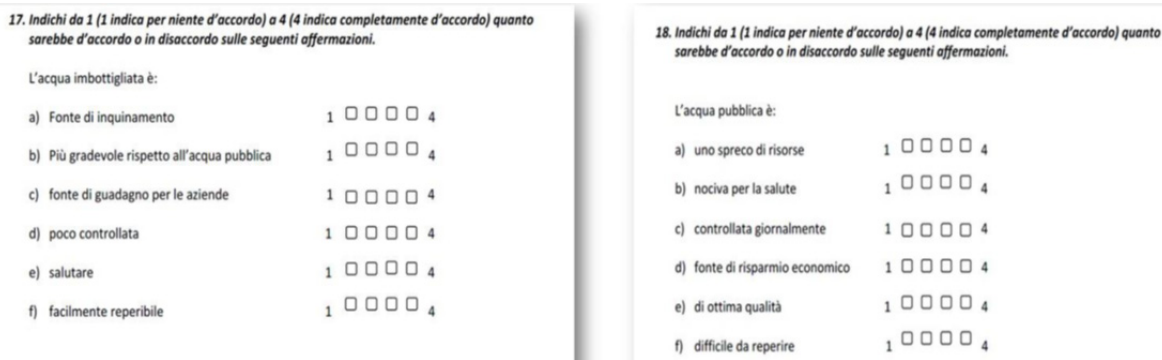

Figura 13: Domande atteggiamento nei confronti dell'acqua pubblica e privata 
Per una maggiore comprensibilità dell'indice costruito su diversi items però, sono state ricodificate le etichette in FAVOREVOLE (ex accordo) e SFAVOREVOLE (ex disaccordo) per rendere la lettura più intuitiva. Nel complesso quindi, dalle interviste effettuate, è emerso che l' $85 \%$ degli intervistati è risultato favorevole all'utilizzo dell'acqua in bottiglia, mentre solo un $15 \%$ sfavorevole (fig. 14).

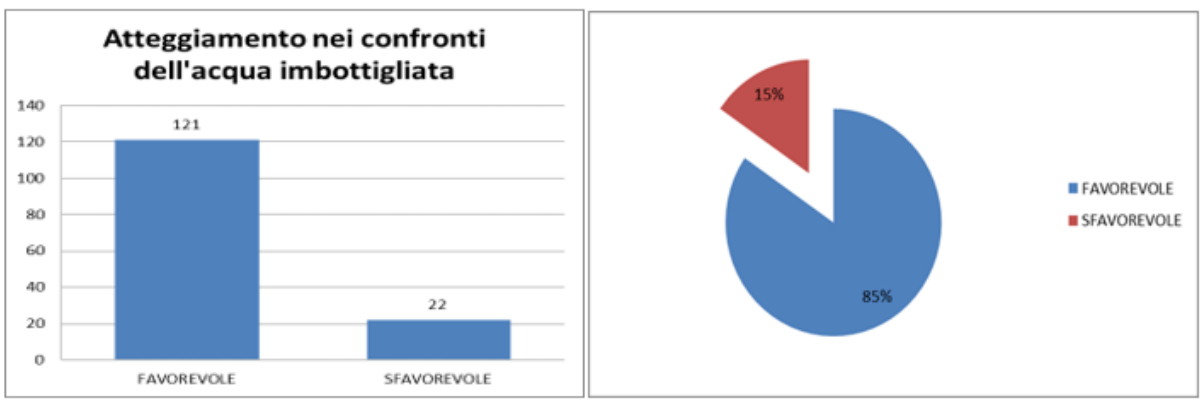

Figura 14: Atteggiamento nei confronti dell'acqua imbottigliata

Per quanto riguarda invece l'utilizzo dell'acqua pubblica, nella maggior parte dei casi gli intervistati sono risultati favorevoli (97\%), e solo il 3\% sfavorevole (fig. 15). Possiamo quindi concludere che, prima dell'esperimento, gli intervistati hanno individuato più aspetti positivi nell'utilizzo di acqua pubblica, piuttosto che nell'acqua in bottiglia.

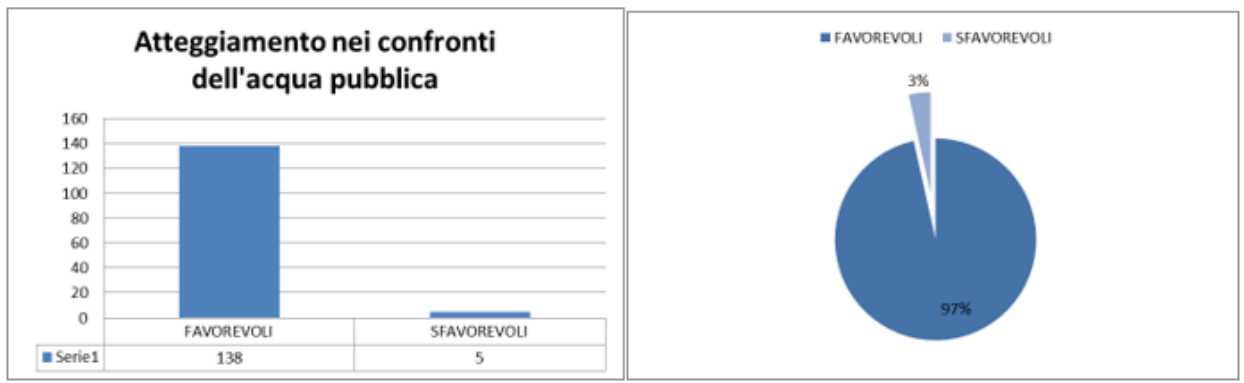

Figura 15: Atteggiamento nei confronti dell'acqua pubblica

\section{Atteggiamento nei Confronti Dell'acqua Pubblica e Privata: Un Esperimento Sociale}

Ai fini del nostro lavoro di seguito illustreremo la qualità dell'acqua presente nella zona di via Salaria ${ }^{3}$, per dimostrare come essa sia stata idonea per lo svolgimento dell'esperimento sociale attuato per verificare la percezione dei consumatori riguardo l'uso di acqua potabile rispetto a quella in bottiglia. I dati raccolti dal sito Acea, gestore dell'acqua a Roma, riportati di seguito, sono il risultato di un'analisi fatta a dicembre 2016.

${ }_{3}^{3}$ Sede del dipartimento di Comunicazione e ricerca sociale, luogo in cui si è svolto l'esperimento sociale. 
Tabella 1: Qualità dell'acqua in via Salaria

\begin{tabular}{|c|c|c|c|}
\hline & Unita di misura & Limiti D. Lgs $31 / 2001$ & Valori medi relativi \\
\hline Conc. ioni idrogeno & $\mathrm{pH}$ & $6,5-9,5$ & 7,54 \\
\hline Cond. Elettrica a $20^{\circ} \mathrm{C}$ & $\mu \mathrm{S} / \mathrm{cm}$ & 2.500 & 563 \\
\hline Bicarbonati & $\mathrm{mg} / \mathrm{HCO} 3$ & & 398 \\
\hline \begin{tabular}{|l|} 
Durezza totale \\
\end{tabular} & ${ }^{\circ} \mathrm{F}$ & $15-50$ & 32,5 \\
\hline Calcio & $\mathrm{mg} / \mathrm{LCa}$ & & 101,2 \\
\hline Magnesio & $\mathrm{mg} / \mathrm{L} \mathrm{Mg}$ & & 17,51 \\
\hline Residuo fisso calcolato & $\mathrm{mg} / \mathrm{L}$ & 1.500 & 403 \\
\hline Cloro residuo libero & $\mathrm{mg} / \mathrm{LCl} 2$ & 0,2 & 0,15 \\
\hline Ammoniaca & $\mathrm{mg} / \mathrm{L} \mathrm{NH} 4$ & 0,5 & $\$ 0,10$ \\
\hline \begin{tabular}{|l|} 
Nitrati \\
\end{tabular} & $\mathrm{mg} / \mathrm{L}$ NO3 & 50 & 2,98 \\
\hline Nitriti & $\mathrm{mg} / \mathrm{L} \mathrm{NO} 2$ & 0,5 & $<0,05$ \\
\hline Clonuri & $\mathrm{mg} / \mathrm{LCl}$ & 250 & 9,1 \\
\hline Fluonuri & $\mathrm{mg} / \mathrm{L}$ & 1,50 & 0,21 \\
\hline Potassio & $\mathrm{mg} / \mathrm{L} \mathrm{K}$ & & 3,75 \\
\hline Sodio & $\mathrm{mg} / \mathrm{L} \mathrm{Na}$ & 200 & 7,0 \\
\hline \begin{tabular}{|l|l|} 
Solfati \\
\end{tabular} & $\mathrm{mg} / \mathrm{L} \mathrm{SO} 4$ & 250 & 17,41 \\
\hline Arsenico & $\mu \mathrm{g} / \mathrm{L}$ As & 10 & $<1,0$ \\
\hline Manganese & $\mu \mathrm{g} n \mathrm{~nL} \mathrm{Mn}$ & 50 & 0,29 \\
\hline
\end{tabular}

Fonte: https://www.acea.it

A partire dai valori relativi alla durezza e ai nitrati e nitriti - che pure contribuiscono a definire la qualità dell'acqua - presenti nell'acqua di via Salaria, risultata potabile, la zona è stata considerata idonea per condurre l'esperimento sociale.

L'esperimento è stato svolto in data 23 novembre 2017, ed è consistito nel far assaggiare agli intervistati le due tipologie di acqua, facendo scegliere se assaggiare acqua liscia o gassata. La strumentalizzazione adottata invece comprendeva quattro brocche di acqua uguali, al cui interno vi erano rispettivamente: acqua liscia pubblica, acqua gassata attraverso un gassificatore pubblica, acqua liscia privata ed acqua gassata privata. Successivamente sono state poste agli intervistati ulteriori domande, al fine di comprendere se gli atteggiamenti nei confronti dell'acqua fossero cambiati dopo l'esperimento.

La figura 16 illustra quanto intervistati hanno notato differenze tra le due acque durante l'esperimento, e quanti sono stati in grado di riconoscere quale fosse l'acqua pubblica e quale quella privata. Si può notare quindi che il $76 \%$ di essi ha affermato di aver riconosciuto una differenza tra le due acque, rispetto ad un $\mathbf{2 2} \%$ che non ha notato differenze. Il $2 \%$ di essi invece si è rifiutato di rispondere. Solo il $52 \%$ degli intervistati però ha dichiarato di essere stato in grado di distinguere le due tipologie di acqua, mentre il $46 \%$ di essi non ne è stato in grado.

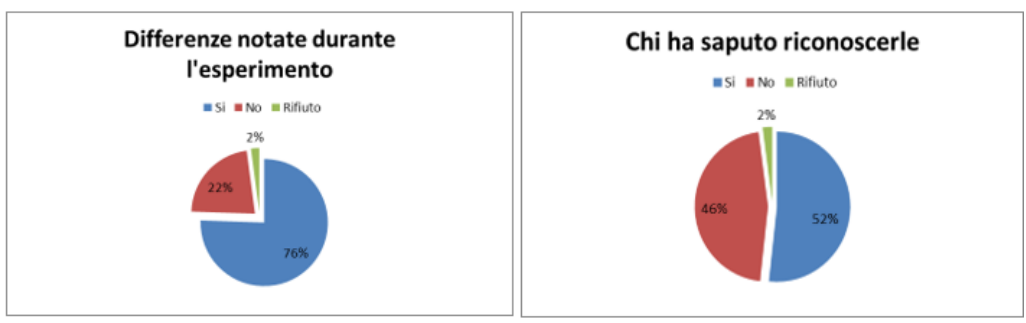

Figura 16: Differenze notate durante l'esperimento e chi ha saputo riconoscerle 
Le figure di seguito illustrano invece l'atteggiamento degli intervistati nei confronti dell'acqua pubblica e privata, prima e dopo l'esperimento. Per ottenere tali risultati, agli intervistati in seguito all'esperimento sono state nuovamente sottoposte le domande utili a comprendere l'atteggiamento nei confronti dell'acqua pubblica e privata, citate precedentemente (fig. 13), al fine di verificare la presenza di eventuali cambiamenti negli atteggiamenti degli intervistati dopo l'esperimento. Osservando la figura 17 si può notare come gli intervistati favorevoli all'acqua pubblica prima dell'esperimento fossero il $97 \%$ e dopo l'esperimento il $94 \%$, quindi non vi è stato un significativo cambiamento dell'atteggiamento dopo l'esperimento.

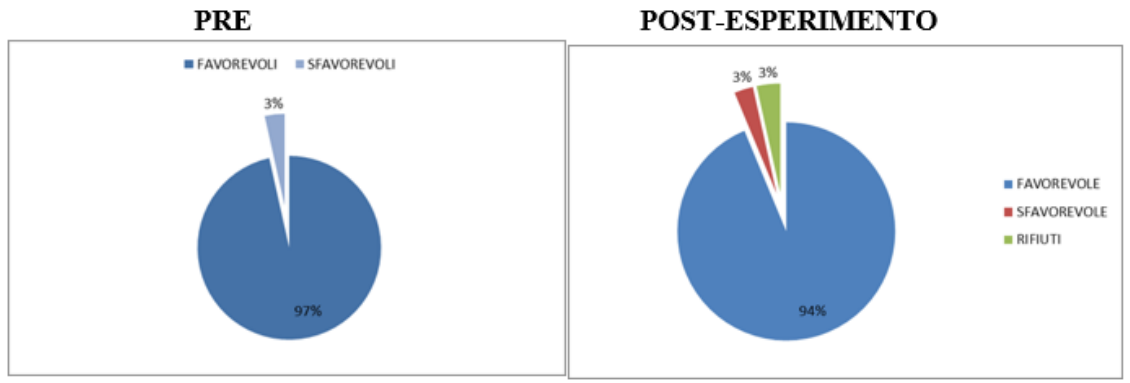

Figura 17 Atteggiamento nei confronti dell'acqua pubblica prima e dopo l'esperimento

La figura 18 illustra in che modo è cambiato l'atteggiamento nei confronti dell'acqua privata dopo l'esperimento. Prima di esso gli intervistati favorevoli erano l'85\% e successivamente sono diventati il $74 \%$, a fronte di un $22 \%$ che dopo l'esperimento si è reso conto della mancanza di differenze tra le due acque e si è detta sfavorevole all'uso di acqua in bottiglia.

\section{PRE}

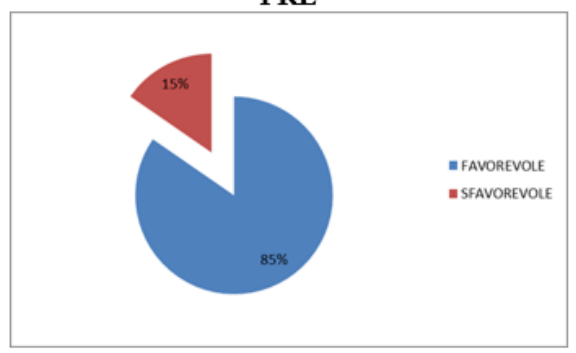

POST-ESPERIMENTO

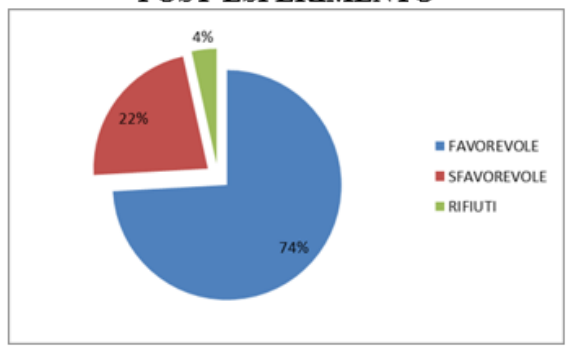

Figura 18 Atteggiamento nei confronti dell'acqua imbottigliata prima e dopo l'esperimento

\section{Conclusioni}

Alla luce dell'analisi e dell'esperimento effettuato quindi, si può concludere che non vi è un'effettiva differenza nelle preferenze tra l'acqua pubblica e privata e l'utilizzo del tipo di acqua utilizzata da parte degli intervistati non dipende da una dimensione culturale, non essendoci una relazione tra la tipologia di acqua utilizzata dagli intervistati e quella utilizzata dalle famiglie. Inoltre, le motivazioni che portano a preferire l'acqua pubblica sono per la maggior parte di tipo economico, considerando anche il campione selezionato, che è composto soprattutto da studenti e infine si può affermare che, non essendovi una correlazione statistica tra l'atteggiamento e l'utilizzo di acqua, la diminuzione dell'atteggiamento positivo nei confronti dell'acqua in bottiglia del $10 \%$ post- esperimento, è imputabile alla buona riuscita di quest'ultimo. 


\section{References}

Al-Salem S.M., Lettieri P., Baeyens J. (2009). Recycling and recovery routes of plastic solid waste (PSW): A review. Waste Management. Volume 29, Issue 10. Pages 2625-2643. Doi: 10.1016/j.wasman.2009.06.004

Cabiddu F. (2007). Comportamento del consumatore e scelte strategiche delle imprese. Milano: Franco Angeli.

Comunità Europea (1998). DIRETTIVA 98/83/CE DEL CONSIGLIO del 3 novembre 1998 concernente la qualità delle acque destinate al consumo umano. Testo disponibile all'indirizzo web: https://eurlex.europa.eu/legal-content/IT/TXT/PDF/?uri=CELEX:31998Loo83

de Mello D., Pezzin S.H., Amico S.C. (2009). The effect of post-consumer PET particles on the performance of flexible polyurethane foams. Polymer Testing. Volume 28, Issue 7. Pages 702-708. Doi: 10.1016/j.polymertesting.2009.05.014

Greenpeace (2021). L'insostenibile peso delle bottiglie di plastica. Testo disponibile all'indirizzo web: https://www.greenpeace.org/static/planet4-italy-stateless/2021/o7/27cdee4e-linsostenibile-peso-dellebottiglie-di-plastica.pdf

Legambiente (2018). Acque in bottiglia 2018 - Un'anomalia tutta italiana. Testo disponibile all'indirizzo web: https://www.legambiente.it/wp-content/uploads/dossier-acque_in_bottiglia_2018.pdf

Parlamento Europeo (2018). Rifiuti di plastica e riciclaggio nell'UE: i numeri e i fatti. Testo disponibile all'indirizzo web: https://www.europarl.europa.eu/news/it/headlines/society/20181212STO21610/rifiuti-di-plastica-ericiclaggio-nell-ue-i-numeri-e-i-fatti

Shah A.A., Hasan F., Hameed A., Ahmed S. (2008). Biological degradation of plastics: A comprehensive review. Biotechnology Advances. Volume 26, Issue 3. Pages 246-265. Doi: 10.1016/j.biotechadv.2007.12.005

Torretta V. (2009). Transportation of dangerous substances: a decisional support system for risk analysis. In: Proceedings of the 2009 International Conference on Chemical, Biological \& Environmental Engineering (CBEE 2009). Singapore 9-11 October, 2009, pp. 310-314. Doi: 10.1142/9789814295048_0063

Torretta V. (2013). Environmental and economic aspects of water kiosks: Case study of a medium-sized Italian town. Waste Management. Volume 33, Issue 5. Doi: 10.1016/j.wasman.2013.01.014

Welle F. (2011). Twenty years of PET bottle to bottle recycling-An overview. Resources, Conservation and Recycling. Volume 55, Issue 11. Pages 865-875. Doi: 10.1016/j.resconrec.2011.04.009 